\title{
Bio-terror Preparedness Exercise in a Mixed Reality Environment*
}

\author{
Alok Chaturvedi, Chih-Hui Hsieh, Tejas Bhatt, and Adam Santone \\ Purdue Homeland Security Institute, Krannert School of Management, 403 West \\ State Street, Purdue University, West Lafayette, IN 47907-2014 \\ \{alok, hsiehc, tejas, santone\}@purdue.edu
}

\begin{abstract}
The paper presents a dynamic data-driven mixed reality environment to complement a full-scale bio-terror preparedness exercise. The environment consists of a simulation of the virtual geographic locations involved in the exercise scenario, along with an artificially intelligent agent-based population. The crisis scenario, like the epidemiology of a disease or the plume of a chemical spill or radiological explosion, is then simulated in the virtual environment. The public health impact, the economic impact and the public approval rating impact is then calculated based on the sequence of events defined in the scenario, and the actions and decisions made during the full-scale exercise. The decisions made in the live exercise influence the outcome of the simulation, and the outcomes of the simulation influence the decisions being made during the exercise. The mixed reality environment provides the long-term and large-scale impact of the decisions made during the full-scale exercise.
\end{abstract}

\section{Introduction}

The Purdue Homeland Security Institute (PHSI) created a Dynamic DataDriven Mixed Reality Environment to support a full-scale bio-terror preparedness exercise. In a mixed reality environment certain aspects of the scenario are conducted in the live exercise, while others are simulated. Actions and outcomes in the live exercise influence the simulated population, and the actions and outcomes of the simulation affect the lessons learned. The simulation modeled the public health aspect of the virtual population, as well as the economy of the virtual geographies. The artificial population would also voice a public opinion, giving a measure of support for the decisions and actions the government is taking on their behalf. The simulation provided the capability to analyze the impact of the crisis event as well as the government response.

With such powerful capabilities, there are numerous advantages to using the simulation to augment the live exercise. The simulation allows us to scale the scenario to a much larger geographical area than possible with just a live exercise, thereby allowing key decision makers to keep the bigger picture in mind.

\footnotetext{
* This research was partially supported by the National Science Foundation's DDDAS program grant \# CNS-0325846 and the Indiana State 21st Century Research and Technology award \#1110030618.
} 
The simulation can execute in faster-than-real-time, allowing the participants to analyze the long-term impacts of their actions in a matter of minutes. The simulation also provides the ability to move forward and backward in virtual time, to analyze possible future implications of current actions, or to go back and retry the response to achieve better results.

In the future we hope to allow the participants to have greater interaction with the simulation. The participants would receive continuously updated statistics from the simulation and the live exercise. This will allow them to make more strategic decisions on the scenario. With hands-on simulation training provided, or technical support staff taking actions on behalf of the participants, more time can be spent analyzing the results of the simulation than dealing with the simulation itself. The simulation is intended to be used as a tool for discussion of critical issues and problems in a response and recovery scenario. With the live exercise and the simulation connecting in real-time, the accuracy of the simulation will greatly improve, thereby providing more meaningful information to the key players.

\section{Computational Modeling}

The computational modeling is based on the Synthetic Environments for Analysis and Simulation (SEAS) platform. SEAS provides a framework that is unbiased to any one specific scenario, model, or system and can be used to represent fundamental human behavior theories without restrictions of what can be modeled common in modern simulation efforts. The enabling technology leverages recent advances in agent-based distributed computing to decouple control as well as data flow. SEAS is built from a basis of millions of agents operating within a synthetic environment. Agents emulate the attributes and interactions of individuals, organizations, institutions, infrastructure, and geographical decompositions. Agents join together to form networks from which evolve the various cultures of the global population. Intricate relationships among political, military, economic, social, information and infrastructure (PMESII) factors emerge across diverse granularities. Statistics calculated from the simulation are then used to provide measurable evaluations of strategies in support of decision making.

The fundamental agent categories in SEAS are the individuals, organizations, institutions, and infrastructure (IOIIG). The population agents of these fundamental types will form higher order constructs in a fractal-like manner, meaning sufficient detail exists at multiple levels of focus, from world constructs to individuals. Higher order constructs include political systems (type of government, political parties/factions), militaries (soldiers, institutions, branches of service), economic systems (formal banking networks and black-market structures), social systems (tribes, religious groups, neighborhoods) and information systems (print, broadcast, internet). Agents representing individuals are used to model the populace in the synthetic environment. Individual agents are categorized into citizen and leader agents. An individual's well being is based on a model consisting of eight fundamental needs: basic, political, financial, security, 
religious, educational, health, and freedom of movement. The desire and perceived level of each of the well being categories are populated taking into account the socio-economic class of the individual the agent represents.

Citizen agents are constructed as a proportional representation of the societal makeup of a real nation. A citizen agent consists of a set of fundamental constructs: traits, well being, sensors, goals, and actions. The traits of citizen agents, such as race, ethnicity, income, education, religion, gender, and nationalism, are configured according to statistics gathered from real world studies. Dynamic traits, such as religious and political orientations, emotional arousal, location, health, and well being, result during simulation according to models that operate on the citizen agents and interactions they have with other agents. The traits and well being determine the goals of a citizen agent. Each citizen agent "senses" its environment, taking into account messages from leaders the citizen has built a relationship with, media the citizen subscribes to, and other members in the citizen's social network. Each citizen agent's state and goals can change as a result of interactions the citizen has with its environment. A citizen agent can react to its environment by autonomously choosing from its repertoire of actions. Additionally, a citizen agent's set of possible actions can change during the course of the simulation, such as when a citizen agent resorts to violence. Traits, well-being, sensors, and actions together determine the behavior of the citizen agent.

Clusters of citizen and leader agents form organizations. Citizen agents voluntarily join organizations due to affinity in perspective between the citizens and the organization. An organization agent's behavior is based on a foundation consisting of the desires of the organization's leaders and members. Organizational leadership constantly seeks maintenance and growth of the organizational membership by providing tangible and intangible benefits, and citizens subscribe based on a perceived level of benefit that is received from the organization. Additionally, through inter-organization networks, attitudes and resources may be shared among organizations. Through these internal and external interactions, organizations cause significant changes in perception and attitude change and become core protagonists of activism in the model. In turn, an organization exercises its power through the control over its resources and its ability to procure and maintain its resource base.

Institution agents are represented as 'governmental entities' such as the army, police, legislature, courts, executive, bureaucracy, and political parties-entities that are able to formulate policies that have legal binding, and have more discretionary resources. SEAS models institutions as structures that are products of individual choices or preferences, being constrained by the institutional structures (i.e. an interactive process). Institutions are like formal organizations with an additional power to influence the behaviors of members and non-members.

Media agents also play a significant role in providing information to other agents in the form of reports on well-being and attitudes. Media organizations consist of television, radio, newspapers, and magazines. The media make choices of what information to cover, who to cover, what statements to report, what 
story elements to emphasize and how to report them. Incidents are framed on well-being components, and formalized in a media report. Media is able to set the agenda for domestic policies as well as foreign policy issues. Citizens subscribe to media organizations based on their ideological bend. Media organizations act primarily to frame the issues for their audiences in such a way that they increase their viewer-ship as well as their influence.

Agents interact with the environment and respond, i.e., take action, to exogenous variables that may be specified by human agents or players in the environment as well as inputs from other agents. This is implemented with the introduction of inputs and outputs that each agent possesses. Inputs consist of general "environmental sensors" as well as particular "incoming message sensors." The incoming message sensors are singled out because of the importance ascribed to each agent to be able to query messages from the environment discriminately. The agent also possesses ports characterized collectively as "external actions" that allow the agent to submit its actions or messages to the environment. Finally, the agent possesses an internal set of rules classified as "internal actions" that result in the agents "external actions" on the basis of the sensor inputs as well as the traits/attributes and intelligence structure of each agent.

\subsection{Virtual Geographies}

The simulation will consist of a fictitious community that contains all the relevant features (hospitals, railways, airports, lakes, rivers, schools, business districts) from any Indiana community. This fictitious community can be customized to mimic a real community within Indiana. The virtual geography may be divided into high population density residential areas, low population density residential areas, commercial areas as well as uninhabitable areas. There can be various level of granularity for different communities as needed for the scenario (from international, national, state, district, county, city to city block levels).

\subsection{Computational Epidemiology of Synthetic Population}

The virtual community will have a virtual population represented by artificial agents. An agent is able to represent the activity of a human through a combination of learned variables and interactions. Research has shown that these agents act as the vertices of a societal network, and that their interactions comprise the edges of the network [Wasserman, 1994]. Like living beings, each agent has different interactions and experiences, and thus acts differently when faced with a situation. And while these evolving differences are essential for a useful simulation, certain predefined traits are also necessary. As an example, though all students in a class may be exposed to a flu virus, certain members will be more susceptible, and case severity will differ among those who contract the illness. For this reason, parameters must be assigned that define the susceptibility of an agent to a given pathogen. The high number of relevant attributes for each agent serves to differentiate each agent from its peers. But as the artificial agents grow in complexity, they must also grow in number, in order to maintain the 
characteristics of the society they seek to describe. Once the society has been sufficiently populated, the artificial agents begin to interact with and learn from each other, forming an environment well suited for analysis and interaction by human agents.

In addition to these behaviors, each agent is endowed with certain characteristics that help to differentiate the population. These attributes help to model the variability in human response to a situation. As an example, a wealthier individual may be more likely to leave a high-risk area, if only because of the financial independence he or she enjoys. The following is a partial list of characteristics that serve to differentiate one artificial agent from another: Age, Sex, Income, Education, and Health.

The decision-making process for an artificial agent is a simplified version of the decision making process of rational humans. When faced with a decision, rational humans consider numerous variables. Using a combination of intuition, experience, and logic, one selects the alternative that leads to a certain goal - usually happiness. And while different decisions vary in magnitude, the underlying cognitive model remains relatively constant. As such, while different physical or psychological needs take precedence in different situations, the human decision-making process can be modeled by considering each need in a hierarchical manner. To illustrate, scholarship has shown that, when presented with a threatening environment, the primary focus of a living being shifts to ensuring its own survival. The list that follows partially describes the variables that an artificial agent considers before making a decision: Security, Information Level, Health, Basic Necessities, Mobility and Freedom, Financial Capability, and Global Economy.

In the SEAS environment, as in the real world, reproductive rates and propagation vary according to the type of disease. Similarly, variables such as population density, agent mobility, social structure, and way of life interact to determine the proliferation of the disease. The government officials, or human agents, interact with the system and control propagation via such means as vaccination, treatment, or agent isolation. The options available to the human agents are the same as in real life, and the effectiveness of these interactions is modeled using statistically verified historical information [Longini, et. al 2000].

\subsection{Public Opinion Model}

While the safety of the artificial agents takes highest precedence, government officials must consider the overall spirit of the population when making decisions. To illustrate, though safety may be maximized by quarantining a city in every instance of potential attack [Kaplan, Craft \& Wein, 2002], such restrictive measures may not be tolerated by the population. To enhance the level of learning they can achieve through the simulation, the human agents must consider the impact on public sentiment that each of their decisions may have. As in real life, each artificial agent determines his or her happiness level using a combination of variables: Current health status - must be alive in order to hold an opinion; perceived security; information level; basic necessities; and freedom of mobility. 


\subsection{Economic Impact Model}

The simulation projects the long term economic impact of the crisis scenario. The economic impact of the crisis scenario as well as the government response is modeled based on the following criteria: Loss from impact on public health, cost of response, loss of productivity, loss of business, and loss from impact on public opinion.

\section{Application: Indiana District 3 Full-Scale Exercise 2007}

For the Indiana District 3 full-scale exercise in January 2007, the crisis scenario involved the intentional release of aerosolized anthrax at a fictitious district-wide festival. The following section describes the district-wide results of the simulation in more detail.

\subsection{Detailed Public Health Statistics for District 3}

As shown in Fig. 1, the scenario assumed that 22,186 people were initially exposed to the anthrax. As the epidemiology of anthrax initiates, 17,693 people who were not exposed to enough anthrax spores move into the recovered health status. The remaining 4,493 people started showing symptoms and falling sick, with a brief period of reduction in symptoms, followed by high fever and an $80 \%$ chance of shock and death. The remaining 892 people eventually recovered. Note: This graph is for the no-intervention case (assuming no mass prophylaxis was started).

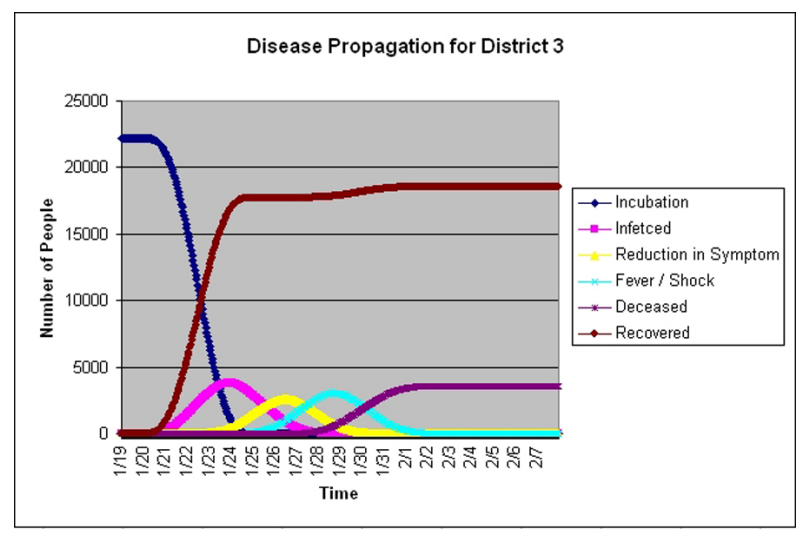

Fig. 1. Disease progression within the population of District 3 over a period of 3 weeks

\subsection{Economic Impact for District 3}

Even with a strong public health response, the District would have to deal with a tremendous economic impact (close to 400 million dollars in the long term) due to the crisis situation, as seen from Fig. 2. 


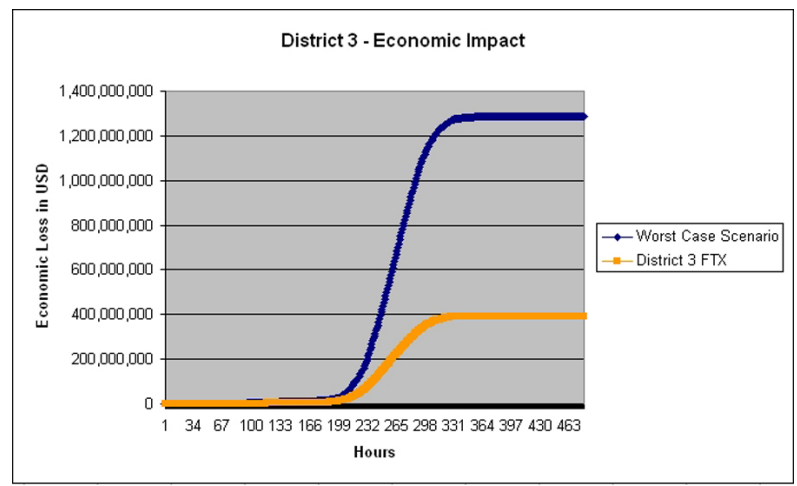

Fig. 2. The economic loss is approximately 900 million dollars less than worst case

\subsection{Public Opinion Impact for District 3}

In the worst case scenario, the population became aware of the crisis when people started to die from the anthrax exposure, as seen in Fig. 3. Hence, public opinion drops at a later date than when the government announces plans for mass prophylaxis. Even though public opinion dropped sooner, it did not go as low as the worst case scenario, due to the proactive and efficient government response.

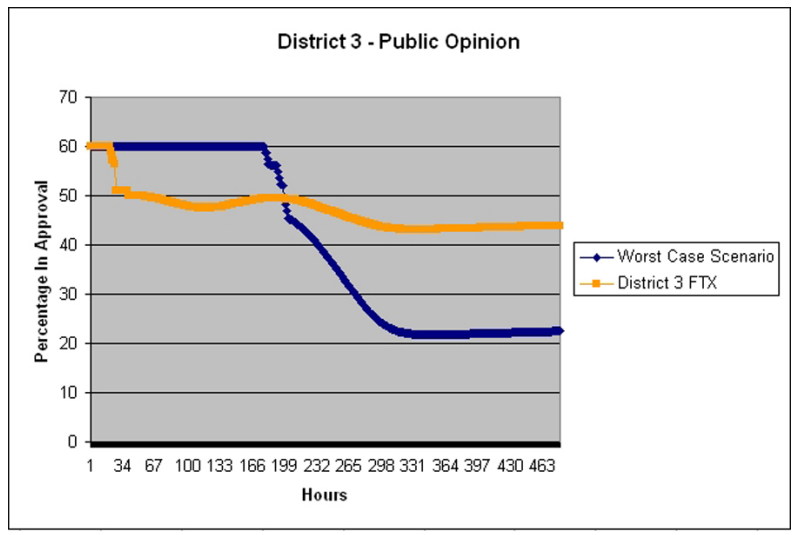

Fig. 3. The public opinion is $21.47 \%$ more in approval of the government than the worst case scenario due to the government response in curbing the situation

\section{Conclusion}

The quick response of the local agencies participating in the exercise resulted in fewer casualties in all counties within District 3 . The counties were quick to 
determine the shortage of regimens available to them locally, and in requesting additional regimens from the state. All the counties would have been able to complete the simulated mass prophylaxis of the exposed population within the timeline guided by the CDC (within 48 hours after exposure) based on their survey responses. This created a dramatic difference (saving 2,505 lives) in the public health statistics of District 3 as compared to the worst case.

While the worst case economic loss would have been around 1.3 billion dollars for District 3, the estimated economic loss for the District 3 Exercise was only 392 million due to the government response and public health actions. Enormous long term economic loss could have crippled the entire district, if the crisis was not handled properly. This situation was avoided during the District 3 Exercise due to positive and efficient government actions.

Initial drop in public opinion was due to the inability of the government to prevent the terror attack from taking place, however, in the long term, the government response and gain of control over the situation, stabilized the public opinion. Based on the public opinion results, it would take some time before the public opinion would come back to normal levels - it likely would take aggressive media campaigns and public service announcements, by the public information officers as well as the elected officials, to mitigate the general state of panic and fear of such an attack happening again.

\section{References}

1. Data from CDC website (http://www.bt.cdc.gov/agent/anthrax/anthrax-hcpfactsheet.asp)

2. Data from FDA website (http://www.fda.gov/CBER/vaccine/anthrax.htm)

3. Reducing Mortality from Anthrax Bioterrorism: Strategies for Stockpiling and Dispensing Medical and Pharmaceutical Supplies. Dena M. Bravata. Table 1

4. Systematic Review: A Century of Inhalational Anthrax Cases from 1900 to 2005 Jon-Erik K. Holty P.g: 275

5. Center for Terrorism Risk Management Policy (http://www.rand.org)

6. The Roper Center for Public Opinion Research at the University of Connecticut

7. E.H. Kaplan, D.L. Craft and L.M. Wein, Emergency response to a smallpox attack: The case for mass vaccination, PNAS, 2002

8. I.M. Longini, M. Elizabeth Halloran, A. Nizam, et al., Estimation of the efficacy of life, attenuated influenza vaccine from a two-year, multi-center vaccine trial: implications for influenza epidemic control, Vaccine, 18 (2000) 1902-1909 